\title{
Magnetic Resonance Imaging of Surgical Implants Made from Weak Magnetic Materials
}

\author{
D. Gogola, A. Krafč́́k, O. Štrbák, I. Frollo \\ Institute of Measurement Science, SAS, Dúbravská cesta 9, SK-841 04 Bratislava, Slovakia, daniel.gogola@savba.sk
}

\begin{abstract}
Materials with high magnetic susceptibility cause local inhomogeneities in the main field of the magnetic resonance (MR) tomograph. These inhomogeneities lead to loss of phase coherence, and thus to a rapid loss of signal in the image. In our research we investigated inhomogeneous field of magnetic implants such as magnetic fibers, designed for inner suture during surgery. The magnetic field inhomogeneities were studied at low magnetic planar phantom, which was made from four thin strips of magnetic tape, arranged grid-wise. We optimized the properties of imaging sequences with the aim to find the best setup for magnetic fiber visualization. These fibers can be potentially exploited in surgery for internal stitches. Stitches can be visualized by the magnetic resonance imaging (MRI) method after surgery. This study shows that the imaging of magnetic implants is possible by using the low field MRI systems, without the use of complicated post processing techniques (e.g., IDEAL).
\end{abstract}

Keywords: NMR imaging, field calculation, metal artifact, surgery implants.

\section{INTRODUCTION}

$\mathrm{I}_{\mathrm{u}}^{\mathrm{v}}$ MAGING OF BIOLOGICAL and physical structures using the magnetic resonance (MR) is a routine investigating procedure today. Special diagnostic techniques have to be applied when an object consisting of weak magnetic materials is inserted into a stationary homogeneous magnetic field of the MR scanner [1]. These materials with higher magnetic susceptibility cause local inhomogeneities of the main field of MR tomograph. These inhomogeneities lead to the loss of phase coherence of the transversal component of the magnetization vector, and thus, rapid loss of image intensity in the area surrounding magnetic implants. Therefore, imaging of tissues adjacent to the magnetic implants is very challenging.

Nevertheless, some materials with low magnetic susceptibility can also cause an artifact in MR images. It may be caused by large and rapidly switched magnetic field gradients which induce eddy currents in electrically conductive sample inserted into the MR scanner. These eddy currents produce additional unwanted, rapidly and slowly decaying magnetic fields [2].

The contrast agents based on the magnetic nanoparticles use the same principle. Signal losses and consequently image intensity decrease cause a negative contrast near the magnetic nanoparticles. Magnetic resonance imaging (MRI) has several advantages over the alternative diagnostics technique. One of the most important advantages is its sensitivity to the subtle differences in the soft tissue structure $[3,4]$. In order to further enhance individual soft tissue, contrast agents are routinely applied. The most commonly used contrast agents are based on nanoparticles of gadolinium or different iron oxides, like $\mathrm{Fe}_{2} \mathrm{O}_{3}$ and $\mathrm{Fe}_{3} \mathrm{O}_{4}$, which are in the form of colloidal suspension in the carrier liquid [5]. Magnetic nanoparticles like polyMAG (Boca Scientific Inc., Florida, USA) can be used for cell labeling. Note that positively charged nanoparticles are readily endocytosed by cells [6]. In the present study, we investigated interaction of an inhomogeneous field induced by the weak magnetic materials with a low field ( 0.18 Tesla) of the whole body clinical MR scanner, benefiting from the fact, that the susceptibility effects are less pronounced at low field MR systems. Our goal was to find the possibility for imaging of selected clinically applied magnetic implants by low field MR tomograph.

\section{SUBJECT \& METHODS}

The stationary magnetic field of MR tomograph has to be highly homogeneous in the selected field of view, with the accepted inhomogeneity limited to the order of $1 \mathrm{ppm}$. If the weak magnetic material with non-zero magnetic susceptibility is placed into the stationary magnetic field $\boldsymbol{B}_{\boldsymbol{0}}$ of the MR tomograph, its uniformity will be disturbed by the additional magnetization $\boldsymbol{J}$. The new value of magnetic field $\boldsymbol{B}$, in the area with insert material, is defined by the following formula (1):

$$
\boldsymbol{B}=\boldsymbol{B}_{0}+\boldsymbol{J}
$$

where:

$$
\boldsymbol{B}_{0}=\mu_{0} \boldsymbol{H} \text { and } \boldsymbol{J}=\chi \mu_{0} \boldsymbol{H}
$$

where:

$\mu_{0}$ - is permeability of vacuum,

$\chi$ - is magnetic susceptibility of inserted material and $\boldsymbol{H}$ - is the intensity vector of stationary magnetic field of the MR tomograph [7].

Magnetic materials in nanoparticle structure are widely used nowadays. Their applications are influenced by their size (hydrodynamic diameter). Depending on their hydrodynamic diameter, they can be divided into two classes, namely superparamagnetic iron oxide (SPIO) nanoparticles, particles with diameter higher than $50 \mathrm{~nm}$, or ultra-small superparamagnetic iron oxide (USPIO) nanoparticles, particles with diameter lower than $50 \mathrm{~nm}$ $[8,9]$.

The critical volume $V_{k}$ of magnetic particles, when particles change their properties from multi-domain to mono-domain, is defined by the following formula: 


$$
V_{k}=\frac{\ln \frac{\tau}{\tau_{0}} \cdot k_{B} \cdot T}{K}
$$

where:

$T$ - is temperature in Kelvin,

$k_{B}-$ is Boltzmann constant,

$\tau$ - is characteristic relaxation time of material,

$\tau_{0}-$ is inverted value of the maximum frequency, at this frequency material of particle reach a maximum change of the magnetic moment,

$K$ - effective uniaxial anisotropy $\left[\mathrm{J} / \mathrm{m}^{3}\right][10]$.

Contrast agents can be characterized by their magnetization $\boldsymbol{J}$ and ${ }^{1} \mathrm{H}$ nuclear magnetic resonance (NMR) relaxation values. Particle size and microstructure have influence on the magnetic and relaxation properties of colloidal suspensions of particles and affect the mechanisms of longitudinal $T_{1}\left(1 / R_{1}\right.$, where $R_{1}-$ is longitudinal relaxation rate) and transverse $T_{2}\left(1 / R_{2}\right.$, where $R_{2}$ - is transversal relaxation rate) proton relaxation times.

Most of clinical MR contrast agents increase the relaxation rate. Contrast agents influence both $T_{1}$ and $T_{2}$ relaxation times, but paramagnetic substances accelerate mainly longitudinal relaxation $T_{1}$, and produce light contrast in $T_{1}$ weighted images. Superparamagnetic substances notably increases the dephasing rate or transverse $T_{2} *$ relaxation $\left(\mathrm{T}_{2}+\right.$ inhomogeneity of the $\boldsymbol{B}_{\boldsymbol{0}}$ field) and then produce dark or negative contrast in $\mathrm{T}_{2}$ weighted images.

Magnetic nanoparticles coated with biocompatible polymers like dextran, polyvinyl alcohol or polyethylene glycol homogeneously diffused in carrier liquid can be applied as intravenous contrast agents. Magnetic nanoparticles together with suitable polymer may be applied into the form of implants of various shapes. One of possible applications is magnetic fibers. Thus, fibers can be used in surgery for internal stitches. These stitches can be visualized by MRI long time after a surgery.

For the purpose of simulation, thirty magnetic nanoparticles with diameters of $35 \mathrm{~nm}$ were arranged in the line. Each nanoparticle is represented by the small current loop. The wire of each loop is imaged like a small dot.

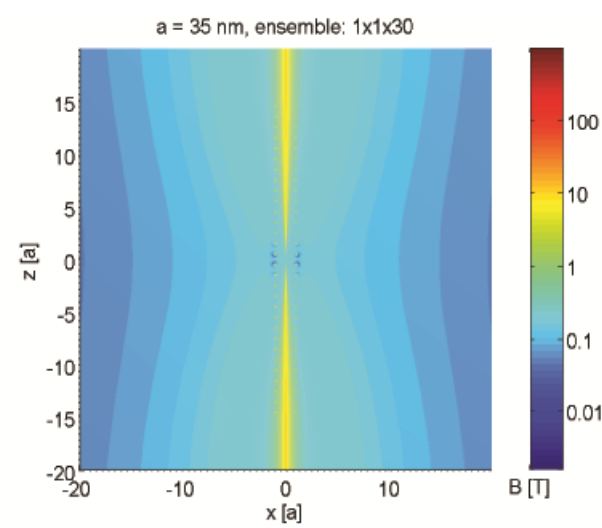

Fig.1. Magnetic field simulation of thirty magnetic nanoparticles, arranged in the line shape.
Magnetic field of such a constellation of magnetic nanoparticles was simulated in Matlab environment (version R2011b, Mathworks Inc., USA) and is shown in Fig.1.

For the simulation of the interaction of stationary magnetic field of the MR scanner and the magnetic nanoparticles, the cubic model equation published in [11] was used.

\section{RESULTS}

For quantification of signal intensity at the ESAOTE Opera open-bore whole body MR scanner (ESAOTE, Genoa, Italy), custom made phantoms were used. These phantoms consisted of distilled water and different concentration of water-based ferrofluid type nanoparticles: EMG 607, made by FerroTec Corporation (FerroTec, Santa Clara, California, USA). Parameters of phantoms are summarized in Table 1.

Table 1. Properties description of the phantoms

\begin{tabular}{|c|c|c|c|}
\hline Sample & $\begin{array}{l}\text { Concentration } \\
\text { EMG607 }[\boldsymbol{\mu g} / \mathbf{m l}]\end{array}$ & $\begin{array}{l}\text { Susceptibility } \\
\text { of sample }\end{array}$ & $\begin{array}{l}\mathbf{T}_{\mathbf{2}} \text { relaxation } \\
\text { time [ms] }\end{array}$ \\
\hline \multirow{2}{*}{$\begin{array}{c}\text { Distilled } \\
\text { water }\end{array}$} & 0 & $-9.04 * 10^{-6}$ & $1800 \pm 20$ \\
\hline \multirow{4}{*}{\begin{tabular}{c} 
Distilled \\
water + \\
\cline { 2 - 4 }
\end{tabular}} & 1.11 & $4.24 * 10^{-5}$ & $31.0 \pm 0.3$ \\
\cline { 2 - 4 } & 2.19 & $3.11 * 10^{-5}$ & $15.2 \pm 0.2$ \\
\cline { 2 - 4 } & 3.26 & $5.58 * 10^{-5}$ & $10.0 \pm 0.08$ \\
\cline { 2 - 4 } & 4.30 & $1.28 * 10^{-4}$ & $8.8 \pm 0.09$ \\
\cline { 2 - 4 } & 5.33 & $1.11 * 10^{-4}$ & $6.4 \pm 0.07$ \\
\cline { 2 - 4 } & 6.33 & $1.33 * 10^{-4}$ & $5.2 \pm 0.05$ \\
\cline { 2 - 4 } & 7.32 & $1.44 * 10^{-4}$ & $4.6 \pm 0.05$ \\
\hline
\end{tabular}

MR image of seven samples with magnetic fluid EMG 607 and one reference (distilled water) shows sequential decrease of intensity of the MR signal, while the concentration of ferrofluid EMG 607 increases (Fig.2.).

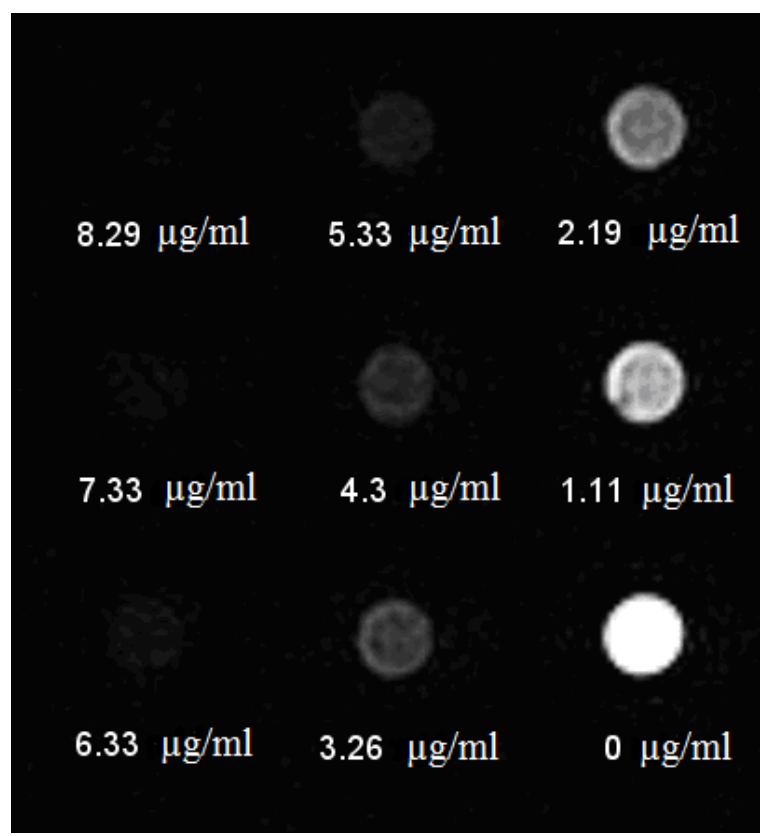

Fig.2. MR image of nine samples with different concentration of EMG607. 
Decrease of signal intensity with dependence on the concentration is shown in Fig.3.

Magnetic susceptibility of each sample was measured by the superconducting quantum interference device (SQUID) at frequency $2.8 \mathrm{~Hz}$ and magnetization field $0.29 \mathrm{mT}_{\mathrm{PP}}$.

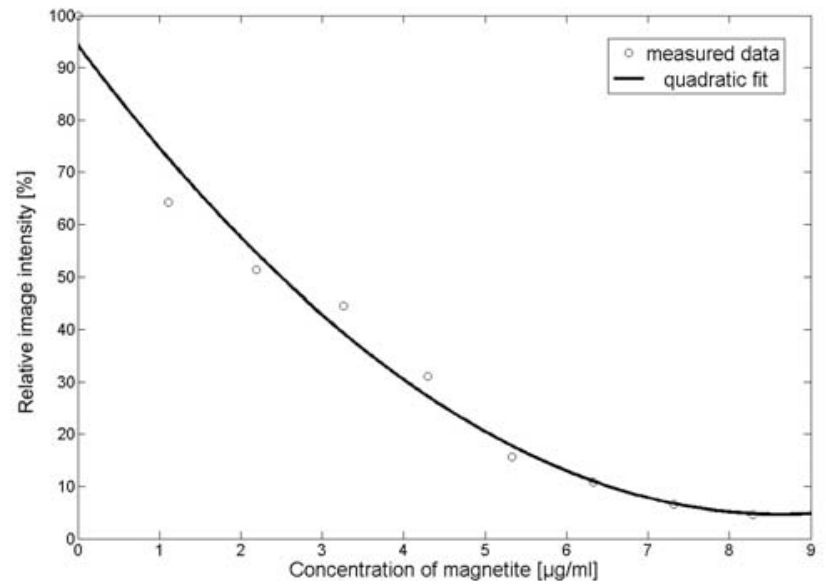

Fig.3. Dependence of relative image intensity on the concentration of magnetite. The distilled water image intensity was used as a reference $(100 \%)$.

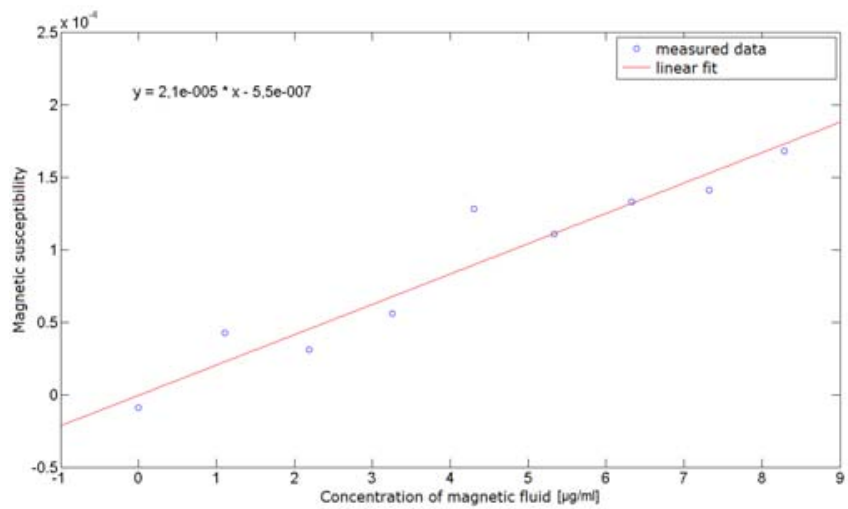

Fig.4. Dependence of magnetic susceptibility on concentration of magnetic nanoparticles in a fluid

The dependence of magnetic susceptibility on concentration of EMG 607 in water is shown in Fig.4.

In the second experiment, we investigated the properties of imaging sequences in order to find the best parameter setup using a planar phantom, as shown in Fig.5. This phantom consisted of four thin strips of magnetic tape, arranged gridwise. Two images of such a phantom were obtained using the Gradient-echo (GRE) sequence with the same setting: echo-time (TE) $10 \mathrm{~ms}$, slice thickness $3 \mathrm{~mm}$ and different TR (left image obtained using TR $240 \mathrm{~ms}$ and right image obtained using TR $800 \mathrm{~ms}$ ).

Fig.6. shows images of the post-operative elbow of the patient after a trauma. For the elbow fixation weak magnetic implants (screws) were used. These images were obtained using the spin-echo (SE) sequence with the following parameters: TE $26 \mathrm{~ms}$, TR $2460 \mathrm{~ms}$, slice thickness $2 \mathrm{~mm}$. Parameters were optimized in order to minimize the susceptibility artifacts.

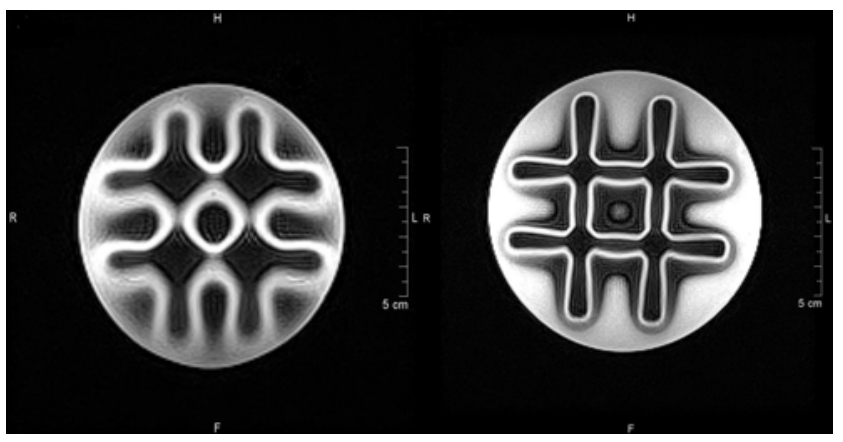

Fig.5. Images of the planar phantom, constructed from strips of magnetic tapes in the shape of grid. Both images were obtained using GRE sequence with the same TE and thickness but different TR. On the left side TR $240 \mathrm{~ms}$ and on the right TR $800 \mathrm{~ms}$.

Fig.7. shows MR images of magnetic fibers consisting of biodegradable polymer and $\mathrm{Fe}_{3} \mathrm{O}_{4}$ nanoparticles. It is one of the typical practical applications of the magnetic nanoparticles. Both images were obtained using the same repetition time $\mathrm{TR}=500 \mathrm{~ms}$, slice thickness $10 \mathrm{~mm}$, but having different echo times TE. Left image in Fig.7. was obtained using $\mathrm{TE}=30 \mathrm{~ms}$ and right image was obtained using $\mathrm{TE}=18 \mathrm{~ms}$.
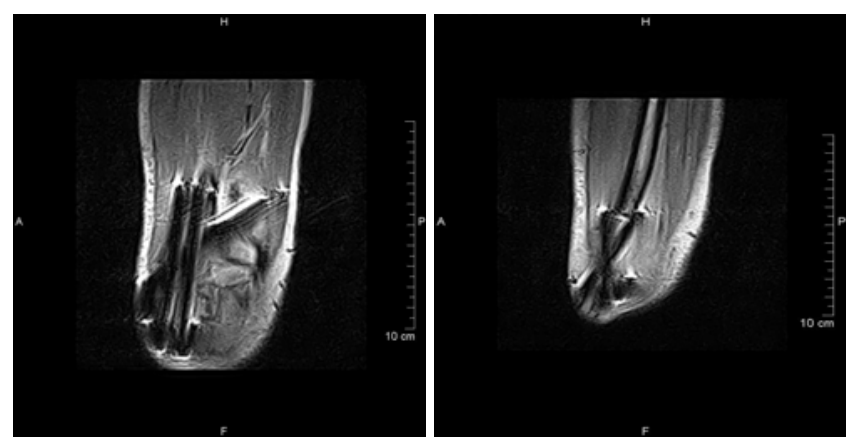

Fig.6. Images of an elbow with magnetic implants. Images were acquired using the SE sequence in sagittal plane.

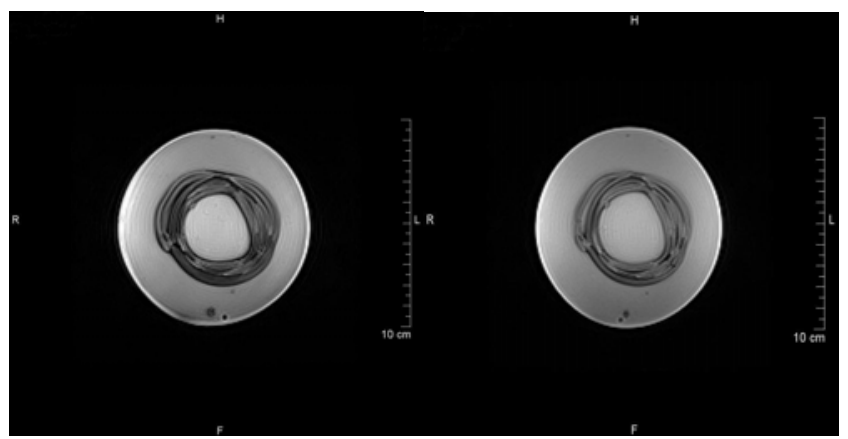

Fig.7. MR Images of magnetic fiber obtained by the GRE sequence using different echo times. 


\section{DISCUSSION / CONCLUSIONS}

The conventional methods which use field mapping to calculate the phase differences between two gradient-echo images, become unreliable because $\mathrm{B}_{0}$ inhomogeneities at high field MRI systems can cause signal dropouts and geometrical distortions in gradient-echo images [12]. For the imaging of the elbow with magnetic implants, spin echo sequence was used. Main reason for that was that the material of the implants had strong magnetic properties in comparison to the weak magnetic phantoms which were tested.

Hundred and nine implants and devices (aneurysm clips, fasteners and staples, coils and stents, heart valve prostheses and annuloplasty rings, orthopedic implants, suture materials, vascular access ports and accessories, miscellaneous implants and devices) were studied in Frank G. Schellok's work [13]. The implants were tested at magnetic field interactions at 3.0 T. This study showed that only $4 \%$ are potentially unsafe based on deflection angle criteria.

Several materials which produce only negligible artifacts within the MR image and their effect on the positional accuracy of features within the image is negligible or can be easily corrected, have been studied in [14]. These types of materials can be considered the magnetic field compatible materials.

Our study shows that imaging of magnetic implants is feasible at low field MR systems $(0.18 \mathrm{~T})$ without the use of any additional post processing techniques (e.g., IDEAL).

\section{ACKNOWLEDGMENT}

This work was supported by the Grant Agency of Slovak Academy of Sciences, VEGA 2/0090/11 and by State program ŠPVV No. 2003SP200280203.

\section{REFERENCES}

[1] Frollo, I., Andris, P., Gogola, D., Přibil, J., Valkovič, L., Szomolányi, P. (2012). Magnetic field variations near weak magnetic materials studied by magnetic resonance imaging techniques. IEEE Transaction on Magnetics, 48 (8), 2334-2339.

[2] Marcon, P., Bartusek, K., Dokoupil, Z., Gescheidtova, E. (2012). Diffusion MRI: Mitigation of magnetic field inhomogeneities. Measurement Science Review, 12 (5), 205-212.
[3] Youtaka, I., Tmohiro, Y. (2005). History, present and future of diagnostic imaging: Magnetic resonance imaging (MRI). Japanisch-Deutsche Medizinische Berichte, 50, 40-51.

[4] Berry, E., Bulpitt, A.J. (2009). Fundamentals of MRI: An Interactive Learning Approach. CRC Press.

[5] Keevil, S. (2001). Magnetic resonance imaging in medicine. Physic Education, 36, 476-485.

[6] Durdík, Š., Babincová, M., Kontrišová, K. Bergemann, C., Babinec, P. (2013). Magnetic nanoparticles as contrast agents for magnetic resonance imaging. The General Science Journal, 1-8, http://gsjournal.net/.

[7] Cicmanec, P. (1980). Basic Physics 2: Electricity and Magnetism. Bratislava: ALFA. (in Slovak)

[8] Bjørnerud, A., Johansson, L. (2004). The utility of superparamagnetic contrast agents in MRI: Theoretical consideration and applications in the cardiovascular system. NMR in Biomedicine, 17, 465-477.

[9] Majewski, P., Thierry, B. (2007). Functionalized magnetite nanoparticles-synthesis, properties and bioapplications. Critical Reviews in Solid State and Materials Sciences, 32, 203-215.

[10] Ma, Z., Liu, H. (2007). Synthesis an surface modification of magnetic particles for application in biotechnology and biomedicine. China Particuology, 5, 1-10.

[11] Štrbák, O., Kopčanský, P., Timko, M., Frollo, I. (2013). Single biogenic magnetite nanoparticle physical characteristics. A biological impact study. IEEE Transactions on Magnetics, 49 (1), 457-462.

[12] Bartusek, K., Dokoupil, Z., Gescheidtova, E. (2006). Magnetic field mapping around metal implants using an asymmetric spin echo MRI sequence. Measurement Science and Technology, 17 (12), 3293-3300.

[13] Schenck, J.F. (1996). The role of magnetic susceptibility in magnetic resonance imaging: MRI magnetic compatibility of the first and second kinds. Medical Physics, 23 (6), 815-851.

[14] Shellock, F.G. (2002). Biomedical implants and devices: Assessment of magnetic field interactions with a 3.0-Tesla MR system. Journal of Magnetic Resonance Imaging, 16, 721-732. 